\title{
Indocyanine Green-Fluorescent Pancreatic Perfusion-Guided Resection of Distal Pancreas in Solid Pseudopapillary Neoplasm: Usefulness and Feasibility During Pancreaticobiliary Surgery
}

\author{
Sung Hyun Kim, M.D., Seoung Yoon Rho, M.D., Chang Moo Kang, M.D., Ph.D. \\ Division of Hepatobiliary Pancreatic Surgery, Department of Surgery, Yonsei University College of Medicine, Pancreaticobiliary Cancer Clinic, Yonsei Cancer \\ Center, Severance Hospital, Seoul, Korea
}

ICG is widely applied in real-time imaging during abdominal surgery, plastic surgery, as well as oncologic staging and treatment. A twenty-eight year-old female patient was found to have a $4.5 \mathrm{~cm}$ solid pseudopapillary neoplasm in the tail of the pancreas. Under ICG-fluorescent pancreatic perfusion-guidance, we easily defined the margin of the pancreatic tumor and secured the resection margin when performing laparoscopic distal pancreatosplenectomy in the patient. No clinically relevant complications, including postoperative pancreatic fistula, were noted. Intravenous ICG can be very easily and quickly detected in the pancreas under near infrared light. This enhanced vision gives strong contrast to the organ compared to a necrotic tumor with poor blood perfusion, such as solid pseudopapillary neoplasm. Based on our current experience, ICG pancreatic perfusion-guided determination of appropriate resection margin is useful and feasible during pancreaticoduodenectomy.

Keywords: Fluorescent dyes, Indocyanine green, Pancreatectomy, Perfusion, Solid pseudopapillary neoplasm
Received July 14, 2017

Revised 1st July 14, 2017

2nd August 3, 2017

3rd August 16, 2017

Accepted August 17, 2017

Corresponding author

Chang Moo Kang

Department of Hepatobiliary and

Pancreatic Surgery, Severance

Hospital, Yonsei University College

of Medicine, 50-1 Yonsei-ro,

Seodaemun-gu, Seoul 03722, Korea

Tel: +82-2-2228-2100

Fax: +82-2-313-8289

E-mail: cmkang@yuhs.ac

ORCID:

http://orcid.org/0000-0002-5382-4658

Copyright $@ 2018$ The Journal of Minimally Invasive Surgery. All rights reserved.
This is an Open Access article distributed under the terms of the Creative Commons Attribution Non-Commercial License (http:/l creativecommons.org/licenses/by-nc/4.0/) which permits unrestricted non-commercial use, distribution, and reproduction in any medium, provided the original work is properly cited.

\section{INTRODUCTION}

Indocyanine green (ICG) is a fluorescent dye that has been approved by the Food and Drug Administration (FDA) for clinical and research use in humans since 1956. ${ }^{1}$ Initially, ICG was used for quantitative measurements of hepatic and cardiac function. ${ }^{2}$ When ICG is injected intravenously, it binds to plasma proteins, such as albumin, and lipoproteins. ${ }^{3}$ It is generally excited between 750 and $800 \mathrm{~nm}$, and fluorescence emission is detected near the maximum peak of $832 \mathrm{~nm}^{4}$ Due to this physiologic property and the advancement of instru- mental camera technology, ICG is now widely applied in realtime imaging during abdominal surgery, plastic surgery, as well as oncologic staging and treatment. ${ }^{5}$ However, there are few examples of clinical applications using ICG in pancreatic surgery. ${ }^{6}$ We recently experienced a very interesting case that supports potential application of ICG in pancreatectomy. 


\section{CASE REPORT}

\section{Patient characteristics}

A 28-year-old female patient visited outpatient department of gastrointestinal internal medicine, and underwent computed tomography (CT) scan. She did not have any past or current medication history. CT showed that the patient had a mass in the tail of pancreas. (Fig. 1A). Pancreaticobiliary endoscopic ultrasonography was also performed, and 44-mm sized eccentric calcification solid mass was presumed as solitary pseudopapillary neoplasm.

\section{Method of ICG injection during surgery}

We used $25.0 \mathrm{mg}$ of DID Indocyanine Green ${ }^{\circledR}$ injection, produced by Doingindang Pharmaceutical. One vial was mixed with $10 \mathrm{cc}$ normal saline and administrated in periph- eral intravenous site. Approximately 3 minutes before confirmation of margin, 2 cc (5 mg of ICG) was injected. The ICG imaging system we used was a product of NOVADAQ Technologies Inc. Under the ICG-fluorescent pancreatic perfusionguidance, we easily defined the margin of pancreatic tumor and secured the resection margin while performing laparoscopic distal pancreatosplenectomy in the patient (Fig. 1B, C).

\section{Pathologic findings}

Final pathologic examination reported that the tumor was a $4.5-\mathrm{cm}$ solid pseudopapillary neoplasm of the pancreas. (Fig. 1D) Distance from resection margin to tumor was reported as $1.5 \mathrm{~cm}$, and the tumor extended beyond the pancreas (pT3). No lymphovascular or perineural invasion was noted. A total of six regional lymph nodes were retrieved, and all of the nodes were free of tumor.
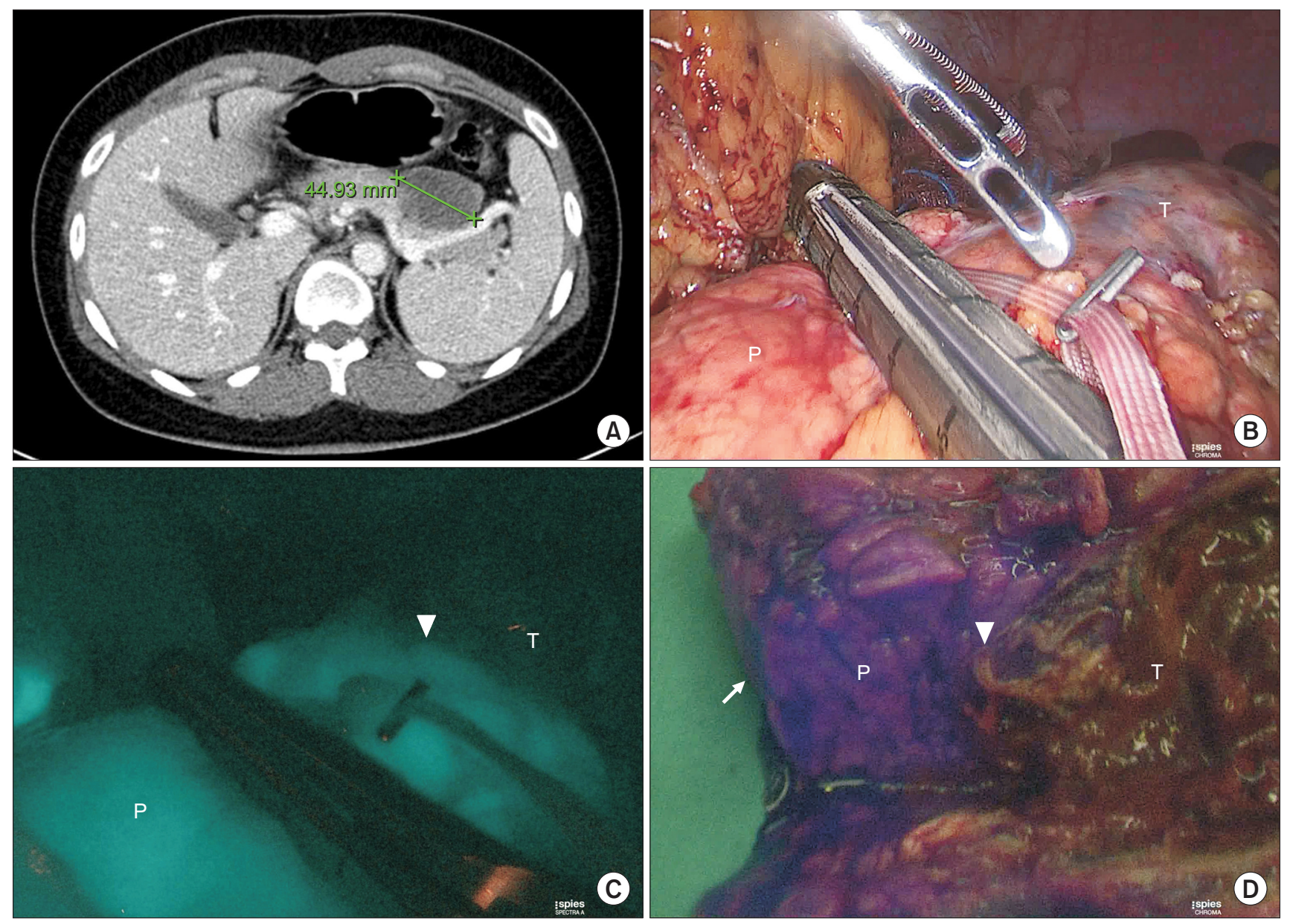

Fig. 1. (A) Preoperative image: $4.5 \mathrm{~cm}$ mass in the pancreas tail. (B) Before pancreas resection (bare eye view), (C) before pancreas resection (ICGfluorescent pancreatic perfusion-guidance view), and (D) Immediate postoperative specimen (ICG-fluorescent pancreatic perfusion-guidance view). $\mathrm{P}=$ pancreas parenchyma; $T$ = tumor; white arrow = resection margin; and white arrowhead = perfusion margin. 


\section{Postoperative recovery}

It was noted that the length of hospital stay was seven days after surgery, during which no significant postoperative complication, such as pancreatic fistula, was reported. Patients received regular follow-ups in outpatient department of surgery.

\section{DISCUSSION}

The pancreas is an internal organ with high blood perfusion, ranging from $38.4 \mathrm{ml} / \mathrm{min} / 100 \mathrm{ml}$ to $356 \mathrm{ml} / \mathrm{min} / 100 \mathrm{ml}^{7}$ Therefore, intravenous ICG can be very easily and quickly detected in the pancreas under near infrared light. This enhanced vision gives strong contrast to the organ compared to a necrotic tumor with poor blood perfusion, such as solid pseudopapillary neoplasm, which surgeons can take advantage of when determining the resection margin of the pancreas. Without this technology, an intraoperative ultrasound or a surgeon's intuition is used for securing the resection margin. In support of this technology, a previous study reported that determining the resection margin using pancreatic perfusion with Doppler techniques was useful in preventing anastomosis site leakage. ${ }^{8}$ Based on our current experience, ICG pancreatic perfusion-guided determination of appropriate resection margin is useful and feasible during pancreaticoduodenectomy.

To the best of our knowledge, this brief report is the first ICG-based real-time intraoperative visualization to determine the appropriate resection margin of the pancreas. Further experiences and clinical applications of ICG-fluorescent-based pancreatic surgery need to be undertaken.

\section{ACKNOWLEDGMENTS}

This research was supported by a grant from National Research Foundation (NRF-2016K1A3A1A12953723).

\section{REFERENCES}

1) Alander JT, Kaartinen I, Laakso A, et al. A review of indocyanine green fluorescent imaging in surgery. Int J Biomed Imaging 2012;2012:940585.

2) Maarek JM, Holschneider DP, Harimoto J, Yang J, Scremin OU, Rubinstein EH. Measurement of cardiac output with indocyanine green transcutaneous fluorescence dilution technique. Anesthesiology 2004;100:1476-1483.

3) Yoneya S, Saito T, Komatsu Y, Koyama I, Takahashi K, DuvollYoung J. Binding properties of indocyanine green in human blood. Invest Ophthalmol Vis Sci 1998;39:1286-1290.

4) Mordon S, Devoisselle JM, Soulie-Begu S, Desmettre T. Indocyanine green: physicochemical factors affecting its fluorescence in vivo. Microvasc Res 1998;55:146-152.

5) Reinhart MB, Huntington CR, Blair LJ, Heniford BT, Augenstein VA. Indocyanine Green: Historical Context, Current Applications, and Future Considerations. Surg Innov 2016;23:166-175.

6) Subar D, Pietrasz D, Fuks D, Gayet B. A novel technique for reducing pancreatic fistulas after pancreaticojejunostomy. J Surg Case Rep 2015;2015.

7) Tsushima Y, Miyazaki M, Taketomi-Takahashi A, Endo K. Feasibility of measuring human pancreatic perfusion in vivo using imaging techniques. Pancreas 2011;40:747-752.

8) Strasberg SM, McNevin MS. Results of a technique of pancreaticojejunostomy that optimizes blood supply to the pancreas. J Am Coll Surg 1998;187:591-596. 